\title{
Influence of static magnetic fields on human myoblast/mesenchymal stem cell co-cultures
}

\author{
CORNELIA EMIKA MUELLER ${ }^{1 *}$, RICHARD BIRK $^{2 *}$, BENEDIKT KRAMER $^{1}$, ANGELA WENZEL $^{1}$, \\ J. ULRICH SOMMER ${ }^{1}$, KARL HÖRMANN $^{1}$, JENS STERN-STRAETER ${ }^{3}$ and CHRISTIAN WEILBACH ${ }^{4}$ \\ ${ }^{1}$ Department of Otolaryngology, Head and Neck Surgery, Medical Faculty Mannheim, \\ University of Heidelberg, D-68167 Mannheim; ${ }^{2}$ Department of Otorhinolaryngology, Head and Neck Surgery, \\ Marburg University Hospital, Philipps-University Marburg, D-35043 Marburg; ${ }^{3}$ HNO-Zentrum Oldenburger Münsterland; \\ ${ }^{4}$ Department of Anesthesiology and Intensive Care Medicine, St. Josefs-Hospital, D-49661 Cloppenburg, Germany
}

Received July 3, 2017; Accepted September 28, 2017

DOI: $10.3892 / \mathrm{mmr} .2017 .8334$

\begin{abstract}
The results of surgical repair of extensive muscle tissue defects are still of primary concern, leaving patients with residual cosmetic and functional impairments. Therefore, skeletal muscle tissue engineering attempts to grow functional neo-tissue from human stem cells to promote tissue regeneration and support defect closure. Despite intensive research efforts, the goal of stable induction of myogenic differentiation in expanded human stem cells by using clinically feasible stimuli, has not yet been reached to a sufficient extent. Therefore, the present study investigated the differentiation potential of static magnetic fields (SMFs), using co-cultures of human satellite cells and human mesenchymal stem cells (MSCs). It has previously been demonstrated that SMFs may act as a promising myogenic stimulus. Tests were performed on co-cultures with and without SMF exposure, using growth medium [high growth factor concentrations (GM)] and differentiation medium [low growth factors concentrations (DM)]. AlamarBlue ${ }^{\circledR}$ assay-based cell proliferation analysis revealed no significant difference between co-cultures with, vs. without SMF stimulation, regardless of growth factor concentrations in the cell culture medium. To determine the
\end{abstract}

Correspondence to: Ms. Cornelia Emika Mueller, Department of Otolaryngology, Head and Neck Surgery, Medical Faculty Mannheim, University of Heidelberg, Theodor-Kutzer-Ufer 1-3, D-68167 Mannheim, Germany

E-mail: emika.mueller@umm.de

Dr Richard Birk, Department of Otorhinolaryngology, Head and Neck Surgery, Marburg University Hospital, Philipps-University Marburg, Baldingerstrasse, D-35043 Marburg, Germany

E-mail: mail@richard-birk.de

*Contributed equally

Key words: tissue engineering, myoblast, satellite cell, mesenchymal stem cell, static magnetic field, differentiation, co-cultures degree of differentiation in co-cultures under stimulation with SMFs, semi-quantitative gene expression measurements of the following marker genes were performed: Desmin, myogenic factor 5, myogenic differentiation antigen 1 , myogenin, adult myosin heavy chain 1 and skeletal muscle $\alpha 1$ actin. In neither GM nor DM was a steady, significant increase in marker gene expression detected. Verifying the gene expression findings, immunohistochemical antibody staining against differentiation markers revealed that SMF exposure did not enhance myogenic maturation. Therefore, SMF treatment of human satellite cell/MSC co-cultures did not result in the desired increase in myogenic differentiation. Further studies are required to identify a suitable stimulus for skeletal muscle tissue engineering.

\section{Introduction}

The Repair of tissue defects following trauma or tumor ablation is one of the major challenges surgeons face today. Especially in anatomical regions where a lesion results in significant impairment of organ function or socially stigmatizing disfiguration, plastic reconstruction with autologous material is appropriate and necessary. The unsatisfactory outcomes of previous attempts to repair muscle defects, e.g., with flaps, triggered the development of alternative treatment approaches, such as tissue engineering (1).

The aim of skeletal muscle tissue engineering is to obtain autologous tissue by isolating and growing stem cells capable of myogenic differentiation. This engineered tissue is then used for tissue reconstruction. However, the induction of complete differentiation in these stem cells is still challenging and virtually only achieved in immortalized cell lines, such as $\mathrm{C} 2 \mathrm{C} 12$ (mouse) or L6 (rat), but not in primary human stem cells (2). Since other studies have shown that human myoblast/mesenchymal stem cell (MSC) co-cultures resulted in an increased degree of differentiation and stimulation with static magnetic fields resulted in enhanced maturation $(3,4)$, the aim of this study was to investigate the effect of statistic magnetic fields (SMFs) on the growth of human myoblast/mesenchymal stem cell (MSC) co-cultures. Satellite cells, also called myoblasts, and human mesenchymal 
stem cells (MSCs) are the preferred stem cells for growing skeletal muscle tissue since their extraction is easily realized by tissue biopsies and are capable of stable myogenic differentiation (5). Their ability to replicate without losing the ability of differentiation is an advantage of MSCs (3), enabling the generation of larger numbers of cells from a smaller population. In addition, MSCs are suitable for autologous grafting and can improve tissue regeneration by means of immunomodulation $(6,7)$. For this reason, combining the two types of stem cells to increase the degree of myogenic differentiation appears to be a promising approach. Studies on MSC/myoblast co-cultures showed a significant increase in myoblast proliferation and up-regulation of the expression of Notch-1, both as mRNA and as protein, indicating myoblast activation (8). Beier et al demonstrated that rats' MSCs/myoblasts form hybrid myotubes as well as an upregulation of the myogenic marker MEF2 (myogenic enhancer factor 2) and $\alpha$-sarcomeric actin, representing indicators of myogenic differentiation in MSCs (3). Since the effects of static magnetic stimulation on myoblasts and MSCs are not yet fully understood and heterogeneous in terms of proliferation and differentiation, depending on cell type and strength of the magnetic field, it is necessary to undertake further studies in this field. Eldashev et al showed that shielding of the earth's magnetic field and thus reduction to $0.3 \mathrm{mT}$ resulted in the inhibition of proliferation and maturation of newborn rat satellite cells, while 60-160 mT magnetic fields had a stimulatory effect (9). Sakurai et al demonstrated that strong SMFs of $10 \mathrm{~T}$ induced the formation of orientated myotubes in immortalized $\mathrm{C} 2 \mathrm{C} 12$ mouse myoblast cell cultures (10). Coletti et al found that in immortalized rat myoblasts (L6) $80 \mathrm{mT}$ SMFs increased the degree of differentiation, resulting in elevated actin and myosin levels and the formation of myotubes (4). However, it was not possible to apply this finding to human myoblasts. Our working group demonstrated that the effect of magnetic stimulation on human myoblasts correlates to the serum concentration in the cell culture medium (11). Myoblasts cultivated in growth medium (GM) under stimulation of SMF showed a higher fusion index, indicative of a higher degree of differentiation, compared to myoblast cell cultures exposed to additional stimulation with a differentiation medium (DM). Likewise, the additional stimulation of human myoblasts with SMFs und hepatocyte growth factor (HGF) did not result in the assumed increase in myogenic differentiation (12). While an increase in marker gene expression in human myoblast cultures under SMF and by adding insulin-like growth factor (IGF) was detected, no contractile skeletal muscle was found (13).

To determine the degree of differentiation in co-cultures under stimulation with SMFs, semi-quantitative gene expression measurements of the following marker genes were performed: myogenic factor 5 (MYF5), myogenic differentiation antigen 1 (MYOD1), myogenin (MYOG), adult myosin heavy chain 1 (MYH1), and skeletal muscle $\alpha 1$ actin (ACTA1). Transcription factor MYF5 is along with MYOD1 and MYOG part of the myogenic regulatory factors. As promoters of numerous muscle-specific genes, they control the fusion of mononucleatd muscle fibers (14). MYF5 promotes myoblast proliferation and is activated together with MYOD1 in the early stage of myogenesis and thus regarded as an early differentiation marker. MYOD1 promotes the exit from the cell cycle and induces myogenesis via positive regulation of cell-cycle inhibitors, such as p21 and $\mathrm{Rb}$. In addition, it inhibits cell cycle activators, such as cyclins and cyclin-dependent kinases (15). MYOG acts at a later stage than MYOD1 and more specificly on the formation of myofibrils (16). The myosin heavy chain (MYH) is a component of the contractile protein myosin, a hexamer consisting of four light chains and two heavy chains. Myosin produces a muscle contraction by transforming chemical energy, derived from the hydrolysis of ATP, into mechanical force. MYH accounts for almost $50 \%$ of the total protein content in skeletal muscle fibers and occurs in at least 10 different isoforms, which are used for the characterization of skeletal muscle fibers in fast-twitch and slow-twitch fibers. During myogenesis, MYH occurs in embryonic, perinatal and adult isoforms. The expression patterns of the MYH isoforms are controlled in a development-specific manner and can thus act as differentiation markers (17). In this study, the adult isoform was used as a differentiation marker. As a further late differentiation marker, ACTA1, a key component of the contractile apparatus, was analyzed. In their studies, Coletti et al showed that in rat myoblasts the stimulation with SMFs resulted in an accumulation of ACTA1 in myotubes (4). For this reason, we conducted proliferation, gene and protein expression studies in human myoblast/MSC co-cultures with and without stimulation by an $80 \mathrm{mT}$ SMF and cultivated in GM and DM to potentially identify a new adequate myogenic stimulus.

\section{Materials and methods}

Cell culture. Following the approval of the Ethics Committee II of the Medical Faculty Mannheim, University of Heidelberg (Mannheim, Germany)-valid for the collection of all cell lines-stem cells were obtained by skeletal muscle biopsies during head and neck surgeries. The biopsy-derived primary human myoblasts were pooled and expanded for three passages. The degree of purity of the satellite culture was determined to be more than $80 \%$ by testing the muscle-specific intermediate filament desmin, using immunohistochemical staining. For myoblast cultivation, cell culture flasks with $0.2 \%$ gelatin coating (culture medium: Ham's F10 Medium $+10 \%$ fetal bovine serum $+2 \mathrm{mM}$ L-glutamine+penicillin/streptomycin/fungizone [PSF]) were used. Cells were cultivated in an incubator at $37^{\circ} \mathrm{C}, 95 \%$ relative humidity and $5 \% \mathrm{CO}_{2}$ in growth medium.

Isolation and cultivation of mesenchymal stem cells from bone marrow. Isolation and cultivation of human mesenchymal stem cells from adult bone marrow of the femoral shaft was carried out as already described by Stern-Straeter (18) by diluting the aspirate with PBS/2 mM EDTA on a Ficoll-Hypaque solution. Cell counting was performed after density gradient centrifugation $(30 \mathrm{~min}$, $435 \mathrm{~g}$, seeded at a concentration of $\left.1 \times 10^{6} \mathrm{cells} / \mathrm{cm}^{2}\right)$ of the mononuclear cell (MNC) fraction (bone marrow monocytic cells). After specification as 'bone marrow-derived fibroblastoid adherent cells', these cells were cultivated in MSCGM 
or DMEM-lg plus 10\% MSC growth supplements. Once confluence between 70 and $90 \%$ was reached, the FACs were cultivated and passaged.

Co-culture of satellite cell cultures and MSCs. The satellite cell cultures (myoblasts) and MSC cultures were mixed in a ratio of 1:1 and cultivated in three cultures in a growth medium (GM) [Ham's F-10, 1\% penicillin/streptomycin/fungizone (PSF), 2 mM L-glutamine (PromoCell GmbH, Heidelberg, Germany) and 10\% fetal bovine serum (PAA Laboratories, Linz, Austria)] or a differentiation medium (DM) [minimal essential medium (PromoCell GmbH), 2\% horse serum (PAA Laboratories), 2 mM L-glutamine, and PSF]. Supernatants were pooled together later. GM and DM were changed every $72 \mathrm{~h}$ and cells were cultivated in an incubator at $37^{\circ} \mathrm{C}, 95 \%$ relative humidity and $5 \% \mathrm{CO}_{2}$ in growth medium.

Use of static magnetic fields for cell stimulation. As described by Coletti et al, a magnetic field of $80 \pm 5 \mathrm{mT}$ was set underneath the cell culture containers (distance cell layer-magnet: $1 \mathrm{~mm}$; magnetic field axial to the magnet's north pole). A control group of cell cultures treated in the same way (see above) was not exposed to this magnetic field.

Immunohistochemistry. The cells cultured on the chamber slides were immunohistochemically characterized to determine the degree of differentiation of the cells with greater accuracy. For this purpose, staining with primary antibodies [peroxidase-producing AB, concentration 1:50 (except for desmin with 1:100, MYH1 1:20) against MYF5, MYOG, desmin, MYH1, and ACTA1] was carried out.

Corresponding biotinylated antibodies were used as secondary antibodies based on a peroxidase reaction and immunologically bound via IgG. For the actual peroxidase reaction, amino-ethylcarbazole (Dako, Glostrup, Denmark) was used as the chromogen.

The slides were washed with PBS and incubated in sheep serum dissolved in PBS for $30 \mathrm{~min}$ at room temperature to prevent unspecific antibody reactions. Harris' hematoxylin was used for counterstaining the cell nuclei. For the final assessment of the immunohistochemical stainings, a Zeiss Axiophot light microscope was used.

Proliferation analysis. The proliferation analysis was carried out using the alamarBlue ${ }^{\circledR}$ assay (Thermo Fisher Scientific, Inc., Waltham, MA, USA). In the first batch, cells were fed with growth medium and differentiation medium and proliferation was measured on the days $0,2,6,8$, and 12 followed by incubation with alamarBlue ${ }^{\circledR}$ for $24 \mathrm{~h}$ and measured via florescence at a wave length of $540 \mathrm{~nm}$. In the second batch, the effect of the SMF was determined. Here, myoblast proliferation was measured under magnetic field stimulation on the days $0,2,6,8$, and 12 .

RNA isolation. In accordance with the manufacturer's instructions, total RNA was isolated, using the RNeasy mini kit (Qiagen GmbH, Hilden, Germany).

RNA concentration, integrity and degree of purity was measured at A260 and A280 nm (A250/A280=1.7-2.0) using the NanoDrop 8000 spectrophotometer (Thermo Fisher
Table I. alamarBlue ${ }^{\circledR}$ proliferation assay results in FU of the of human MSC/MB co-cultures on GM and DM without SMF.

\begin{tabular}{lccccc}
\hline & Day 0 & Day 2 & Day 6 & Day 8 & Day 12 \\
\hline GM & 15.87 & 17.09 & 21.86 & 28.36 & 44.29 \\
DM & 15.87 & 21.79 & 23.17 & 28.19 & 49.27 \\
\hline
\end{tabular}

FU, fluorescence unit; MSC, mesenchymal stem cell; MB, myoblast; GM, growth medium, high growth factor concentrations; DM, differentiation medium, low growth factors concentrations; SMF, static magnetic field.

Scientific, Inc.) and the Agilent 2100 bioanalyzer (Agilent Technologies, Inc., Santa Clara, CA, USA).

cDNA synthesis and semi-quantitative PCR. For cDNA synthesis, total RNA was used and treated with 1 U DNAse for $30 \mathrm{~min}$ at $37^{\circ} \mathrm{C}$. Reverse transcription of the RNA $(0.5 \mu \mathrm{g})$ was carried out using the oligo(dT)-primed first-strand cDNA synthesis kit (Roche Diagnostics GmbH, Mannheim, Germany) for $1 \mathrm{~h}$ at $42^{\circ} \mathrm{C}$. Using Taq DNA polymerase (Amersham Pharmacia Biotech, Buckinghamshire, UK) and using 2-5 $\mu \mathrm{l}$ from each reverse-transcription products, all cDNA samples were tested for the following genes: MYOG, ACTA1, MYF5, MYOD1, desmin, MYH1, and GAPDH. Therefor a Primus 96 Plus thermal cycler (MWG Biotech, Freiburg, Germany) was used.

Electrophoresis and analysis. Electrophoresis was run in $2 \%$ agarose gel with added ethidium bromide. Subsequently, images of the PCR products were displayed under UV light. Using GAPDH as a standard, relative gene expression was calculated with the software ImageJ (National Institutes of Health, Bethesda, MD, USA).

\section{Results}

Proliferation analysis from satellite cell/MSC co-cultures with and without static magnetic field (SMF) stimulation. The proliferation behavior was determined using the alamarBlue ${ }^{\circledR}$ proliferation assay from day 0 to day 12 in human satellite cell/MSC co-cultures, cultivated in growth medium (GM) or differentiation medium (DM). In addition, these co-cultures were stimulated with SMFs. Cell cultures without SMF stimulation served as controls. The proliferation behavior of the co-culture showed in both GM and DM without SMF stimulation continuously increasing proliferation rates. For details see Table I. The measured fluorescence units (FUs) increased from a baseline value of 15,81 at the start of the cell culture to values of 21,66 in GM and 24.92 in DM on day 6 to values of 41.77 on day 12 in GM and 49.75 in DM. Thus, FUs in DM-treated co-culture were at all points slightly above those measured in GM-cultivated co-cultures. Under additional stimulation with SMFs, no significant change in proliferation rates neither in DM-cultivated co-cultures nor in GM-treated co-cultures was overserved. In this group as well, the proliferation rate showed a steady increase. On day 6, FUs of 21.86 were measured in 


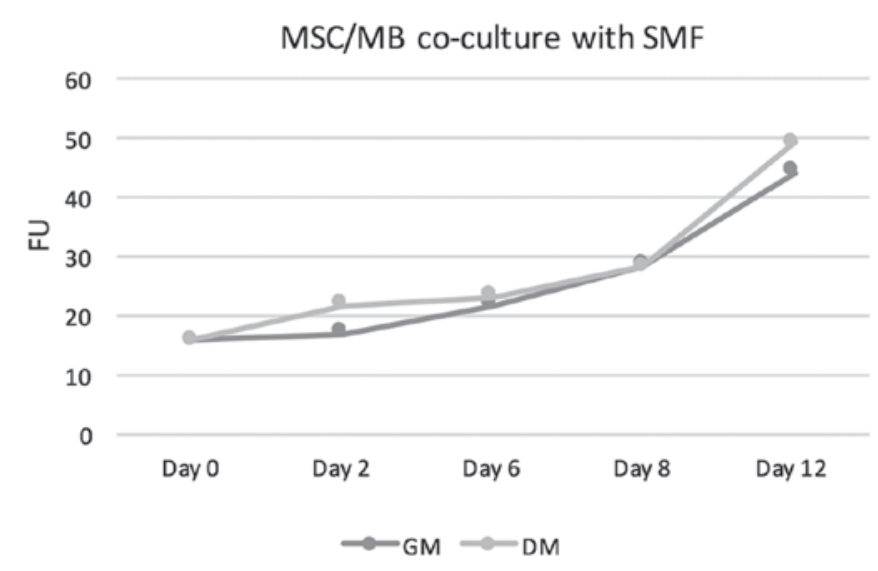

Figure 1. alamarBlue ${ }^{\circledR}$ proliferation assay of human mesenchymal stem cells (MSC)/myoblast (MB) co-cultures on growth medium (GM) and differentiation medium (DM) without static magnetic field (SMF). FU, fluorescence unit.

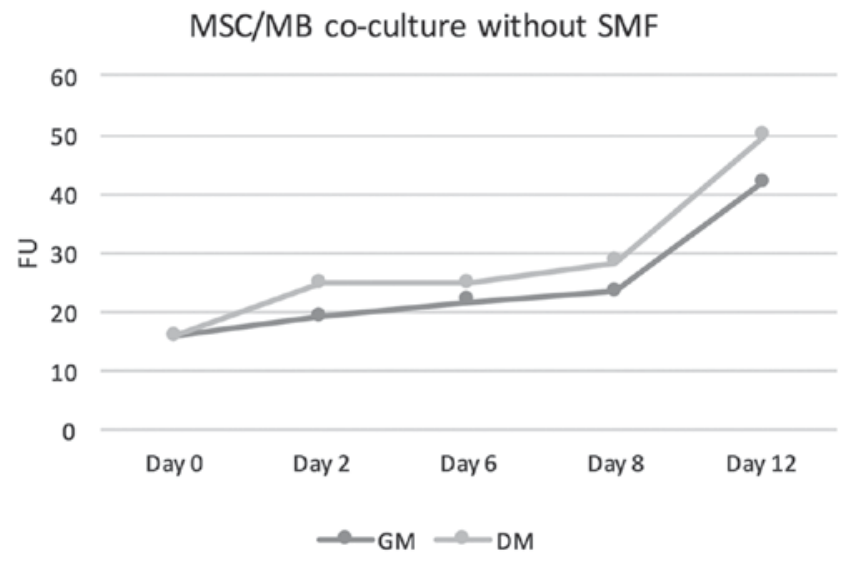

Figure 2. alamarBlue ${ }^{\circledR}$ proliferation assay of human mesenchymal stem cells (MSC)/myoblast (MB) co-cultures on growth medium (GM) and differentiation medium (DM) with static magnetic field (SMF) exposure. FU, fluorescence unit.

GM+SMF and FUs of 23.17 in DM+SMF. On day 12, the FUs were 44.29 in GM+SMF and 49.27 in DM+SMF. Figs. 1 and 2 provides graphical interpretation.

\section{Gene expression analysis}

MYF 5. Gene expression analysis of the early myogenic differentiation marker MYF5 showed positive findings in all tested co-cultures. On day 2, the relative expression values, both in GM and in DM, were lower in the co-cultures stimulated with SMF compared to the non-stimulated cultures. On day 6 and on day 8 , however, the relative expression rates in co-cultures treated with SMF, both in GM and in DM, were above those in non-stimulated co-cultures. On day 12, MYF5 expression of non-stimulated co-cultures was slightly higher in GM, whereas in DM a significantly higher expression rate was found in stimulated cells. For graphical interpretation see Fig. 3.

MYOD1.As showninFig.3, during the firstdays of the cellculture, MYOD1 expression analysis showed initially low expression rates, both in SMF-stimulated and non-SMF-stimulated co-cultures. On day 2 and day 6, the expression rates found in both SMF-stimulated and non-SMF-stimulated co-cultures were almost identical, regardless of the culture medium. On day 8, relative expression of MYOD1 significantly increased in SMF-stimulated co-cultures cultivated in DM. The highest expression rates were detected on day 12 . Here, relative expression in co-cultures growing in GM were higher in SMF-stimulated cells compared to non-SMF-stimulated cells. In DM-cultivated co-cultures, by contrast, higher expression rates were detected in non-SMF-stimulated cells. In the gene expression analysis of MYOD 1, the initially similarly low values are noteworthy, then, strikingly, there was a high value for cell proliferation in the differentiation medium under the influence of the magnetic field on day 8 , while on day 12 these values were below those in the growth medium with and without SMF and in the DM without SMF.

MYOG. Gene expression measurement of myogenin showed a mild time-dependent increase in all groups examined; the highest expression was detected on day 12 in SMF-stimulated co-cultures cultivated in DM. For details see Fig. 3.

ACTA1. On day 2, ACTA1 expression initially showed slightly lower values for SMF-stimulated co-cultures compared to the non-SMF-stimulated groups. On days 6, 8 and 12, however, the expression values of SMF-treated cultures, both in GM and DM cultivated cells, were above those in non-SMF-stimulated cells. The highest expression was detected on day 12 in SMF-stimulated co-cultures cultivated in DM (Fig. 3).

MYH1. Gene expression measurement of MYH1 as a terminal differentiation marker showed significantly higher expression values on day 8 compared to days 2 and 6 . Non-SMF-stimulated co-cultures demonstrated higher expression rates compared to SMF-stimulated ones. On day 12, however, MYH1 expression in stimulated co-cultures was higher compared to non-stimulated co-cultures cultivated in GM. In SMF-stimulated co-cultures cultivated in DM, MYH1 expression was markedly depressed (Fig. 3).

Desmin. Expression analysis of desmin showed a continuous rise in expression with increasing cultivation length. Starting from day 6, the expression values of SMF-stimulated co-cultures were higher compared to those of non-stimulated cells, regardless of the cell culture medium used (Fig. 3).

Immunohistochemistry. To validate mRNA measurements and to identify conceivable differences to the protein form, immunohistochemical stainings using monoclonal antibodies against desmin, MYOD1, MYOG, and ACTA1 were performed. Stain distribution is shown in GM at Table II and in DM in Table III. Fig. 4 provides examples of immunohistochemical stainings.

Desmin. Immunohistochemical staining of MSC/satellite cell co-cultures to the muscle-specific intermediate filament desmin was detected at all points in time in both GM and DM cultivated cells. In co-cultures cultivated with GM, SMF stimulation did not result in any difference in expression rate until day 8 . On day 12, the proportion of desmin measured in non-SMF-stimulated co-cultures was higher compared to the 


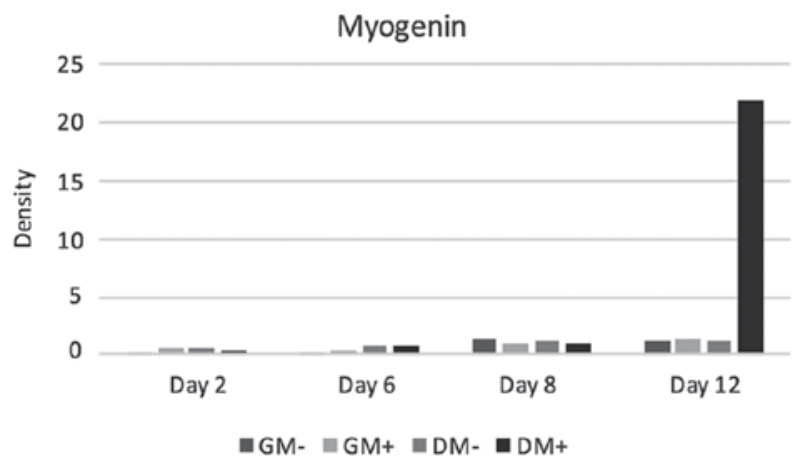

MYH1
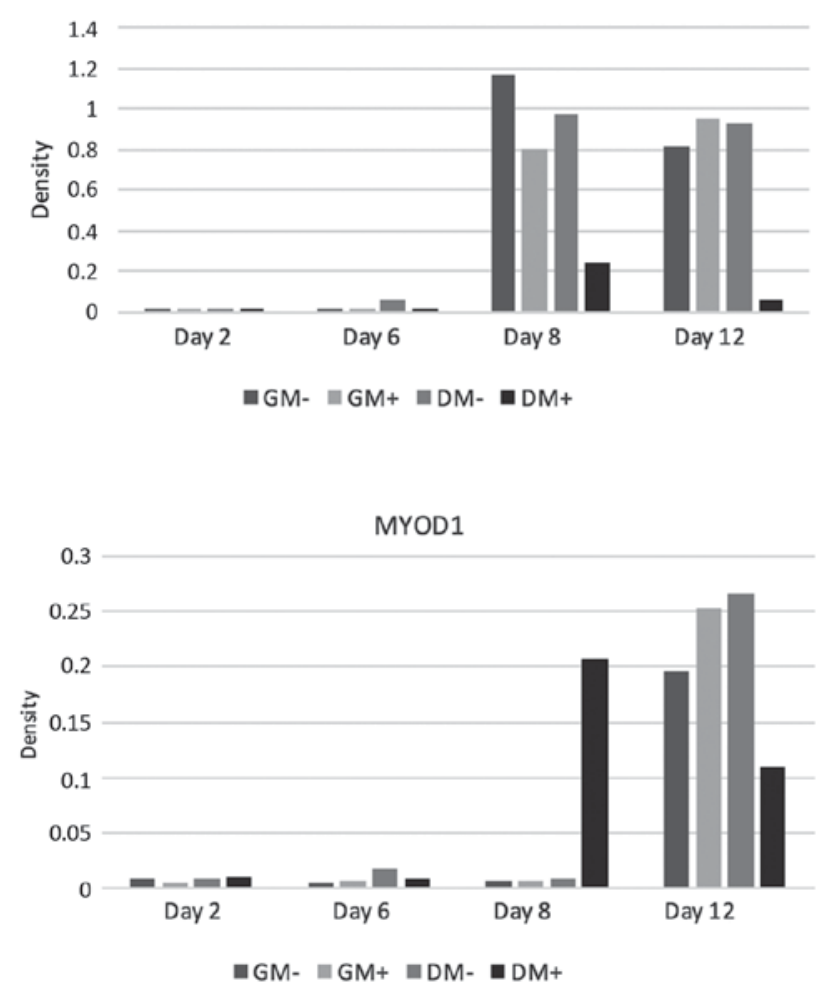

Desmin

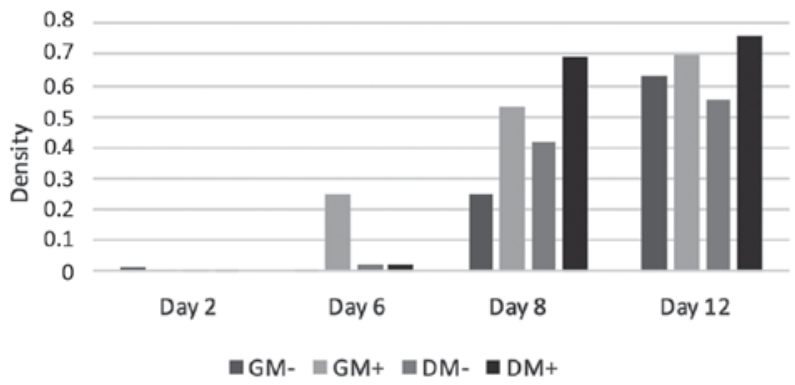

MYF5

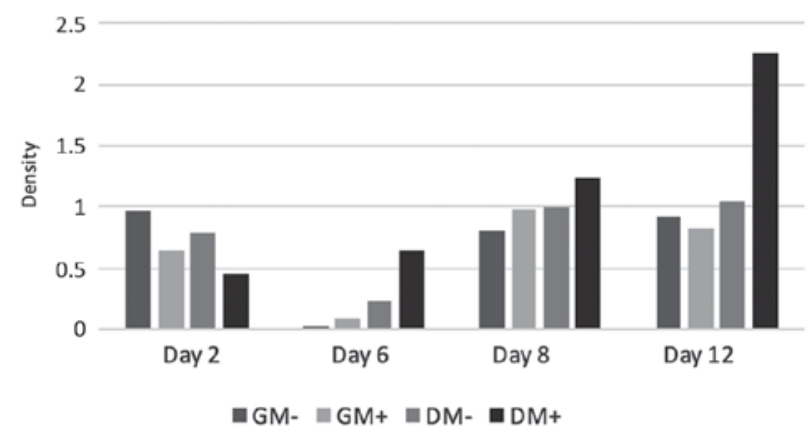

ACTA1

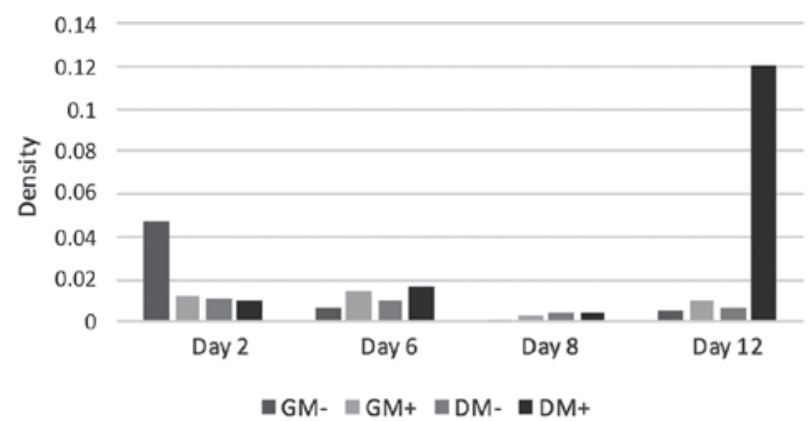

Figure 3. Relative gene expression analyses of MYF5, MYOD1, ACTA1, MYH1, Desmin and myogenin in human mesenchymal stem cells (MSC)/myoblast (MB) co-cultures on growth medium (GM) and differentiation medium (DM) with (+) and without (-) static magnetic field (SMF) exposure. GAPDH served as a reference gene.

stimulated co-cultures. By contrast, in cultures cultivated with DM, no difference between stimulated and non-stimulated co-cultures was detected.

MYOG. Immunohistochemical staining to the transcription factor MYOG in co-cultures cultivated in GM revealed a trend towards higher detection rates in non-SMF-stimulated cultures compared to stimulated cultures. Without SMF stimulation, the detection rates on days 2 and 6 were higher than on days 8 and 12. In DM, myogenin as an early differentiation marker was only detected on the first days of cell culture, regardless of with or without SMF stimulation.

ACTA1. As a component of the contractile apparatus, the late differentiation marker ACTA 1 was detected in non-SMF-stimulated cell cultures cultivated in GM, starting from day 2 of the cell culture. The highest detection rates in this group were achieved on day 12. Under the influence of SMFs, ACTA1 detection in the GM group declined and only on day 6 expression rates were the same. At all other times, the detection rates were below those found for non-SMF-stimulated MSC/satellite cell co-cultures. ACTA1-positive cells were reduced by using DM and only detectable without SMF stimulation on days 2 and 6. With additional SMF stimulation, ACTA1 was solely detected on day 12 .

\section{Discussion}

Induction of stable myogenic differentiation in human stem cells is a basic requirement for skeletal muscle tissue engineering intended to generate adequate amounts of tissue for the repair of skeletal muscle defects, resulting from injuries or tumor ablation procedures. Given their muscle origin and stable myogenic differentiation potential, satellite cells are 
Table II. Stain distribution of Desmin, Myogenin and ACTA1 in human MSC/(MB) co-cultures on GM with and without SMF exposure. ${ }^{\mathrm{a}}$

GM without SMF

GM with SMF

\begin{tabular}{|c|c|c|c|c|c|c|c|c|c|c|}
\hline \multirow[b]{2}{*}{ Antibodies } & & \\
\hline & Day 0 & Day 2 & Day 6 & Day 8 & Day12 & Day 0 & Day 2 & Day 6 & Day 8 & Day 12 \\
\hline Desmin & ++ & + & ++ & ++ & ++ & ++ & + & ++ & ++ & + \\
\hline Myogenin & ++ & ++ & ++ & +-++ & +-++ & ++ & neg & ++ & + & + \\
\hline ACTA1 & neg & + & + & + & +-++ & neg & + & neg & + & \\
\hline
\end{tabular}

'Percentage of cells stained with monoclonal antibodies shown as follows: ‘++++' (70-100\%), ‘+++' (50-70\%), '++' (30-50\%), ‘+' (1-30\%) and 'neg' (no staining). GM, growth medium, high growth factor concentrations; DM, differentiation medium, low growth factors concentrations; MSC, mesenchymal stem cell; MB, myoblast; SMF, static magnetic fields; ACTA1, skeletal muscle $\alpha 1$ actin.

Table III. Stain distribution of Desmin, Myogenin and ACTA1 in human MSC/MB co-cultures on DM with and without SMF exposure. ${ }^{\mathrm{a}}$

DM without SMF

DM with SMF

\begin{tabular}{lccccccccccccc}
\cline { 2 - 7 } Antibodies & Day 0 & Day 2 & Day 6 & Day 8 & Day 12 & & Day 0 & Day 2 & Day 6 & Day 8 & Day 12 \\
\hline Desmin & ++ & + & + & + & + & & ++ & + & & + & + \\
Myogenin & ++ & ++ & neg & neg & neg & ++ & ++ & + & neg & neg \\
ACTA1 & neg & + & + & neg & neg & neg & neg & neg & + & + \\
\hline
\end{tabular}

aPercentage of cells stained with monoclonal antibodies shown as follows: ‘++++' (70-100\%), ‘+++' (50-70\%), ‘++' (30-50\%), ‘+' (1-30\%) and 'neg' (no staining). GM, growth medium, high growth factor concentrations; DM, differentiation medium, low growth factors concentrations; MSC, mesenchymal stem cell; MB, myoblast; SMF, static magnetic fields; ACTA1, skeletal muscle $\alpha 1$ actin.

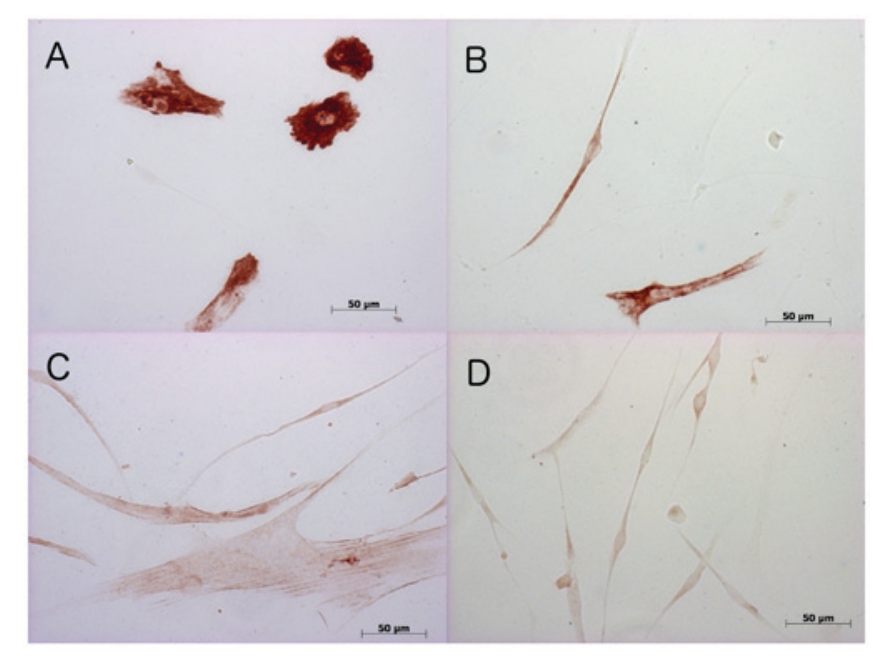

Figure 4. Example of the immunohistochemical staining with/without static magnetic fields (SMF) exposure of (A) Desmin on day 2 in differentiation medium (DM) with SMF; (B) Desmin on day 2 in DM without SMF; (C) Myogenin on day 2 in DM with SMF; and (D) Myogenin on day 2 in DM without SMF.

the most promising and most often used primary cells for the cultivation of skeletal muscle (19). However, satellite cells loose their differentiation ability. Therefore, the production of large volumes of muscle tissue sufficient to meet today's clinical demand is still a very challenging task (20). One reason for the loss of differentiation ability is the heterogeneity in the satellite cell population (21).

Mesenchymal stem cells (MSCs) are regarded as an alternative, promising cell type, because they do not loose their differentiation potential following expansion (3) and can be extracted from a variety of tissue types, including bone marrow, adipose tissue, umbilical cord blood, and placental tissue. However, whether all types of MSCs or only subpopulations can be differentiated into skeletal muscle, remains unclear $(18,22,23)$. The phenotype and myogenic differentiation potential of the different MSCs vary with the respective tissue from which the cells originate (24). It was shown that MSCs from bone marrow were capable of supporting muscle regeneration in vivo $(5,25)$ and thus appeared to be suitable for tissue engineering. However, attempts to achieve myogenic differentiation of human MSCs of bone marrow origin solely by stimulation with cell culture media failed (18). Thus, it was assumed that paracrine factors, such as cytokines, and the extracellular matrix play an important role in the process of myogenic differentiation. Another way to accomplish myogenic differentiation of MSCs is to grow them in co-culture with satellite cells. Beier et al showed that rat MSCs in co-culture with myoblasts formed myotubes (3). Likewise, Di Rocco et al demonstrated that a co-culture combining adipose mouse MSCs and myoblasts boosted the myogenic phenotype (26). However, there is a lack of studies investigating human satellite cell/MSC co-cultures, even though such data are crucial 
for tissue engineering. To gain a better understanding, we conducted this study. Static magnetic fields are another myogenic differentiation stimulus that has the potential to be clinically useful. For example, Coletti et al showed that in the L6 rat cell line SMFs promoted actin and MYH1 formation, indicative of increased differentiation (4). However, studies with human satellite cells, the preferred stem cell for skeletal muscle tissue engineering, found that the effect of SMF stimulation depends on the growth factor concentration in the cell culture medium and that a combination of differentiation medium (low growth factor concentration) and SMF did not result in the desired increase in the degree of differentiation (11). For this reason, it is of interest to investigate the effect of SMF stimulation on human MSC/satellite cell co-cultures and to assess its impact on myogenic differentiation potential.

To determine the effect of SMFs on proliferation behavior in co-cultures, alamarBlue ${ }^{\circledR}$ cell proliferation assays were performed. These showed that $80 \mathrm{mT}$ SMF stimulation had no effect on proliferation behavior in these co-cultures, regardless of the growth factor concentration in the cell culture medium. This result is in line with our data from human satellite cell cultures. It is also confirmed by data obtained from myoblast cultures derived from other species, showing that SMFs of this strength do not influence the proliferation behavior of the cells studied $(4,11)$. That the proliferation rate in DM were slightly higher compared to those of the co-cultures in GM, is an unexpected finding, since in cultures with only satellite cells the high growth-factor concentration resulted in increased proliferation (11). Apparently, this effect does not occur in human MSC/satellite cell co-cultures-a new insight. Given the continued proliferation of MSCs cultured under low growth factor conditions, MSC proliferation capacity is apparently to some extent independent of growth factor concentrations in the cell culture medium used. This phenomenon appears to offset the inhibited proliferation capacity of human satellite cells, as the proliferation measurements in MSC/satellite cell co-cultures yielded comparable proliferation rates for growth medium and differentiation medium (high and low growth factor concentrations). This confirms the results of our previous studies where we showed that the percentage of growth factor in cell culture medium had no significant effect on the proliferation capacity of human MSCs derived from adipose tissue or bone marrow (18). Analysis of quantitative gene expression measurements of the early myogenic marker genes MYF5, MYOD1 and myogenin revealed a rise in expression rates in the co-culture with advancing cell culture duration. The highest expression rates of MYF5, MYOD1 and myogenin were detected on day 12. However, neither in co-cultures cultivated in GM nor in those cultivated in DM, a repeated effect of SMF stimulation was detectable. At all points of measurement, the muscle, specific intermediate filament desmin, which, due to its early expression during myogenesis, is an early myogenic marker (27), was detected. As with MYF5, MYOD1 and myogenin, the highest expression rates were detected on day 12 . This shows that myogenic differentiated cells were present in the co-cultures at all points in time and that the degree of differentiation increased with time. However, no evidence of a significant, continuous effect of SMF stimulation, independent of growth factor concentrations in the cell culture medium, was found.
For myogenic markers indicative of late myogenesis, such as ACTA1 and MYH1, increased expression values were measured during the later days of cell culture monitoring. However, for these markers too, no significant effect of SMFs on myogenic differentiation behavior, in terms of an increase in marker gene expression, was detected. Therefore, the cells of the co-culture do undergo myogenic differentiation with advancing cell culture duration, but this differentiation process is not enhanced by SMF exposure, regardless of the growth factor concentration in the cell culture medium. Consequently, the results obtained for SMF stimulation of human satellite cell monocultures are not consistent with those obtained for human MSC/satellite cell co-cultures. For monocultures we demonstrated that the SMF-induced pro-myogenic stimulation effect was dependent on growth factor concentration (11). We found that only cultures grown in GM showed increased fusion as an indicator of myogenic maturation, but not satellite cells cultured in DM. Since we could not demonstrate this effect in the co-culture, it represents a new research finding. While the exact mechanism underlying the effect of SMFs remains unclear, we know that it is influenced by cell type and cell origin. Contrary to our expectations, this study did not find a pro-myogenic effect of SMFs in human MSC/satellite cell co-cultures. Another factor which may explain the difference between our results and those of Beier et al is their use of rat myoblasts and rat MSCs as well as other stimulating agents (basic fibroblast growth factor; dexamethasone) (3). This shows that the results obtained in studies using cells from other species cannot always be applied to human stem cells-an insight which is of fundamental importance for tissue engineering.

Overall, the analysis revealed marked heterogeneity in the expression rates of the analyzed markers. This can be explained by the fact that MSCs represent a heterogeneous group of cells with diverse myogenic differentiation potential. Similarly, the studies by Di Rocco et al found significant variability in the analyzed myogenic markers (26). The results of the quantitative gene expression measurements are partly confirmed by the results of the immunohistochemical examinations. Immunohistochemical staining succeeded in detecting myogenic markers in the co-culture, regardless of the growth factor concentration in the cell culture medium. However, here again, SMF stimulation did not result in any significant increase in myogenic markers, such as desmin and ACTA1. Overall, immunohistochemical staining showed high variability in the measurements of the myogenic markers which is explained by the heterogeneity of the MSCs.

In conclusion, $80 \mathrm{mT}$ SMF stimulation had no pro-myogenic effect on human satellite cell/MSC co-culture, regardless of the growth factor concentrations in the cell culture medium.

\section{Acknowledgements}

This study contains parts of the doctoral thesis of Cornelia Emika Müller.

\section{References}

1. Stern-Straeter J and Hörmann K: New perspectives in skeletal muscle tissue engineering. HNO 62: 415-422, 2014 (In German). 
2. Stern-Straeter J, Riedel F, Bran G, Hörmann K and Goessler UR: Advances in skeletal muscle tissue engineering. In Vivo 21: 435-444, 2007.

3. Beier JP, Bitto FF, Lange C, Klumpp D, Arkudas A, Bleiziffer O, Boos AM, Horch RE and Kneser U: Myogenic differentiation of mesenchymal stem cells co-cultured with primary myoblasts. Cell Biol Int 35: 397-406, 2011.

4. Coletti D, Teodori L, Albertini MC, Rocchi M, Pristerà A, Fini M, Molinaro M and Adamo S: Static magnetic fields enhance skeletal muscle differentiation in vitro by improving myoblast alignment. Cytometry A 71: 846-856, 2007.

5. Ferrari G, Cusella-De Angelis G, Coletta M, Paolucci E, Stornaiuolo A, Cossu G and Mavilio F: Muscle regeneration by bone marrow-derived myogenic progenitors. Science 279: 1528-1530, 1998.

6. Garcia-Castro J, Trigueros C, Madrenas J, Pérez-Simón JA, Rodriguez R and Menendez P: Mesenchymal stem cells and their use as cell replacement therapy and disease modelling tool J Cell Mol Med 12: 2552-2565, 2008.

7. Pittenger MF and Martin BJ: Mesenchymal stem cells and their potential as cardiac therapeutics. Circ Res 95: 9-20, 2004.

8. Sassoli C, Pini A, Chellini F, Mazzanti B, Nistri S, Nosi D, Saccardi R, Quercioli F, Zecchi-Orlandini S and Formigli L: Bone marrow mesenchymal stromal cells stimulate skeletal myoblast proliferation through the paracrine release of VEGF. PLoS One 7: e37512, 2012.

9. Eldashev IS, Shchegolev BF, Surma SV and Belostotskaia GB: Effect of low-intensity magnetic fields on the development of satellite muscle cells of a newborn rat in the primary culture. Biofizika 55: 868-874, 2010 (In Russian).

10. Sakurai T, Hashimoto A, Kiyokawa T, Kikuchi K and Miyakoshi J: Myotube orientation using strong static magnetic fields. Bioelectromagnetics 33: 421-427, 2012.

11. Stern-Straeter J, Bonaterra GA, Kassner SS, Faber A, Sauter A, Schulz JD, Hörmann K, Kinscherf R and Goessler UR: Impact of static magnetic fields on human myoblast cell cultures. Int J Mol Med 28: 907-917, 2011.

12. Birk R, Sommer U, Faber A, Aderhold C, Schulz JD, Hörmann K, Goessler UR and Stern-Straeter J: Evaluation of the effect of static magnetic fields combined with human hepatocyte growth factor on human satellite cell cultures. Mol Med Rep 9: 2328-2334, 2014.

13. Birk R, Sommer JU, Haas D, Faber A, Aderhold C, Schultz JD, Hoermann K and Stern-Straeter J: Influence of static magnetic fields combined with human insulin-like growth factor 1 on human satellite cell cultures. In Vivo 28: 795-802, 2014.

14. Christ B and Brand-Saberi B: Limb muscle development. Int J Dev Biol 46: 905-914, 2002.

15. Brand-Saberi B: Genetic and epigenetic control of skeletal muscle development. Ann Anat 187: 199-207, 2005.
16. Ridgeway AG, Petropoulos H, Wilton S and Skerjanc IS: Wnt signaling regulates the function of MyoD and myogenin. J Biol Chem 275: 32398-32405, 2000.

17. Pette D and Staron RS: Myosin isoforms, muscle fiber types, and transitions. Microsc Res Tech 50: 500-509, 2000.

18. Stern-Straeter J, Bonaterra GA, Juritz S, Birk R, Goessler UR, Bieback K, Bugert P, Schultz J, Hörmann K, Kinscherf R and Faber A: Evaluation of the effects of different culture media on the myogenic differentiation potential of adipose tissue- or bone marrow-derived human mesenchymal stem cells. Int J Mol Med 33: 160-170, 2014.

19. Stern-Straeter J, Bran G, Riedel F, Sauter A, Hörmann K and Goessler UR: Characterization of human myoblast cultures for tissue engineering. Int J Mol Med 21: 49-56, 2008

20. Carlson ME and Conboy IM: Loss of stem cell regenerative capacity within aged niches. Aging Cell 6: 371-382, 2007.

21. Pietrangelo T, Puglielli C, Mancinelli R, Beccafico S, Fanò G and Fulle S: Molecular basis of the myogenic profile of aged human skeletal muscle satellite cells during differentiation. Exp Gerontol 44: 523-531, 2009.

22. Dezawa M, Ishikawa H, Itokazu Y, Yoshihara T, Hoshino M, Takeda S, Ide C and Nabeshima Y: Bone marrow stromal cells generate muscle cells and repair muscle degeneration. Science 309: 314-317, 2005

23. Corti S, Strazzer S, Del Bo R, Salani S, Bossolasco P, Fortunato F, Locatelli F, Soligo D, Moggio M, Ciscato P, et al: A subpopulation of murine bone marrow cells fully differentiates along the myogenic pathway and participates in muscle repair in the $\mathrm{mdx}$ dystrophic mouse. Exp Cell Res 277: 74-85, 2002.

24. de la Garza-Rodea AS, van der Velde-van Dijke I, Boersma $H$ Gonçalves MA, van Bekkum DW, de Vries AA and Knaän-Shanzer S: Myogenic properties of human mesenchymal stem cells derived from three different sources. Cell Transplant 21: 153-173, 2012.

25. LaBarge MA and Blau HM: Biological progression from adult bone marrow to mononucleate muscle stem cell to multinucleate muscle fiber in response to injury. Cell 111: 589-601, 2002.

26. Di Rocco G, Iachininoto MG, Tritarelli A, Straino S, Zacheo A, Germani A, Crea F and Capogrossi MC: Myogenic potential of adipose-tissue-derived cells. J Cell Sci 119: 2945-2952, 2006.

27. Allen RE, Rankin LL, Greene EA, Boxhorn LK, Johnson SE, Taylor RG and Pierce PR: Desmin is present in proliferating rat muscle satellite cells but not in bovine muscle satellite cells. J Cell Physiol 149: 525-535, 1991. 